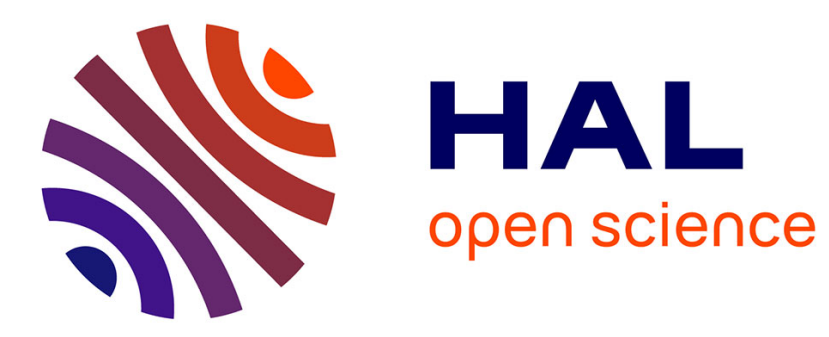

\title{
Nonlocal effects in sand flows on an inclined plane
} F. Malloggi, Bruno Andreotti, E. Clément

\section{To cite this version:}

F. Malloggi, Bruno Andreotti, E. Clément. Nonlocal effects in sand flows on an inclined plane. Physical Review E: Statistical, Nonlinear, and Soft Matter Physics, 2015, 91 (5), pp.052202. 10.1103/PhysRevE.91.052202 . hal-01187780

\section{HAL Id: hal-01187780 \\ https://hal.science/hal-01187780}

Submitted on 17 Nov 2015

HAL is a multi-disciplinary open access archive for the deposit and dissemination of scientific research documents, whether they are published or not. The documents may come from teaching and research institutions in France or abroad, or from public or private research centers.
L'archive ouverte pluridisciplinaire HAL, est destinée au dépôt et à la diffusion de documents scientifiques de niveau recherche, publiés ou non, émanant des établissements d'enseignement et de recherche français ou étrangers, des laboratoires publics ou privés. 


\title{
Nonlocal effects in sand flows on an inclined plane
}

\author{
F. Malloggi, ${ }^{*}$ B. Andreotti, and E. Clément \\ Laboratoire de Physique et Mécanique des Milieux Hétérogènes (PMMH), UMR 7636 CNRS-ESPCI-Universités Paris 6 et 7 , \\ 10 rue Vauquelin 75231 Paris Cedex 05, France \\ (Received 22 November 2014; published 18 May 2015)
}

\begin{abstract}
The flow of sand on a rough inclined plane is investigated experimentally. We directly show that a jammed layer of grains spontaneously forms below the avalanche. Its properties and its relation with the rheology of the flowing layer of grains are presented and discussed. In a second part, we study the dynamics of erosion and deposition solitary waves in the domain where they are transversally stable. We characterize their shapes and velocity profiles. We relate their translational velocity to the stopping height and to the mass trapped in the avalanche. Finally, we use the velocity profile to get insight into the rheology very close to the jamming limit.

DOI: 10.1103/PhysRevE.91.052202

PACS number(s): 45.70.Ht, 47.57.Gc, 83.80.Fg
\end{abstract}

\section{INTRODUCTION}

\section{A. Nonlocal rheology}

Gravity-driven particulate flows are not only a central issue in many geophysical processes but also of great concern in a variety of technological domains involving chemical, pharmaceutical, food, metallurgical, or construction industries. The crucial difficulty to overcome to bring a coherent and useful conceptual vision of these flows still lies in a poor understanding of the passage between flow and blockade. In earth processes, these questions pertain as a missing piece necessary to provide a full understanding in many situations such as dune migration [1], landslides [2-4], snow avalanches [5], underwater gravity currents [6], and coastal geomorphology [7,8], which also are encumbered with many unresolved questions around the mechanics of the dense solid fraction motion and its possible mobilization by a particulate flow.

A first step towards a quantitative description of dense granular flows was achieved after a comprehensive survey of granular flows in a variety of experimental settings and conditions performed by various groups was conducted [9]. This article has popularized the so-called "local" constitutive relation of dense granular flows, first introduced by Refs. [10,11], which is based on dimensional analysis. In a homogeneous shear flow, both the volume fraction $\phi$ and the ratio of the shear stress $\tau$ to the normal stress $P$, a ratio which can be interpreted as an effective friction coefficient, are functions of a single dimensionless parameter, the inertial number $I$ [9]. Denoting the shear rate by $\dot{\gamma}$, the density by $\rho$, and the mean grain diameter by $d$, the inertial number compares the mean shear rate to a reorganization time controlled by $P$ :

$$
I=\frac{\dot{\gamma} d}{\sqrt{P / \rho}}
$$

Equivalently the squared inertial number $I^{2}$ compares the inertial stress $\rho \dot{\gamma}^{2} d^{2}$ to the confining pressure $P$. This local rheology $\tau / P=\mu(I)$ provides a quantitative framework of dense flows, far enough from jamming. The scalar rheology has

\footnotetext{
${ }^{*}$ Present address: LIONS CEA, Saclay, France.
}

been extended to a tensorial form by Ref. [12] and successfully tested on a granular flow confined between rigid walls, away from jamming conditions. Nonlocality refers to any deviation to a local constitutive law $\tau / P=\mu(I)$. For instance, the kinetic theory, valid for dilute, rapid granular flows, is nonlocal since it introduces mean squared velocity fluctuations (the so-called granular temperature) as an independent field controlling the stress tensor [13].

In the case of dense granular flows, it was first proposed that nonlocality results from the distant transmission of momentum through "force chains" [14-16]. To take into account the observation that nonlocality is enhanced in the vicinity of jamming, other mechanisms have recently been proposed, based either on mechanical activation of plastic events [17-21] or on the contact network topology [22]. Different models, not prejudging any microscopic mechanisms, were proposed to capture macroscopically the nonlocal effects [23-29]. Independently of the nature of the dynamical mechanism underlying nonlocality, the existence of a "dynamic" phase transition between a fluid state and a solid state, where the word "dynamic" refers here to the fact that structural symmetries do not change across the transition, allows one to formulate Ginzburg-Landau models for the rheology. The three models proposed so far differ by the choice of the order parameter and of the control parameter. In the partial fluidization theory $[23,25]$, the control parameter is the stress ratio $\tau / P$, and the order parameter characterizes the relative fraction of solid-like behavior and liquid-like behavior in the rheology. In the fluidity theory $[26,27]$, the control parameter is also $\tau / P$, and the order parameter, called the fluidity, is defined as the ratio of the shear rate $\dot{\gamma}$ to the stress ratio $\tau / P$. The third theory [29] is based on a [30] gradient expansion of the rheology with respect to the inertial number $I$. Contrarily to the two others, it does not consider the shear stress as a state parameter. The present article takes a step aside with respect to this series of articles to underline the differences between the nonlocal behavior of rough sand grains and of smooth glass beads.

\section{B. Flow on an inclined plane}

Experiments of smooth, nearly spherical beads have been widely used to extract the rheology of sheared granular assemblies in the inclined plane geometry [9]. An inclined plane can be considered as a rheometric device where the ratio 
of the shear stress to the normal stress $\tau / P=\tan (\theta)$ is fixed everywhere in the bulk of the flow. In this situation, the local rheology predicts that the inertial number $I$ is selected by the plane angle $\theta$ according to the relation $\mu(I)=\tan \theta$. One immediately deduces a velocity profile of the form

$$
u(y)=\frac{2}{3} I \frac{(g \cos \theta)^{1 / 2}}{d}\left[h^{3 / 2}-(h-y)^{3 / 2}\right],
$$

called Bagnold's profile. Numerical simulations using ideal grains have evidenced deviations from this law close to the free surface and close to the bottom. These deviations become dominant when approaching, for a given inclination angle $\theta$, the stopping height $h_{\text {stop }}(\theta)$. Linear or even sublinear profiles have then been observed.

Experimentally it is difficult to access the velocity profile in the bulk. Previous experiments have therefore focused on the mean flow velocity $\bar{u}$ and on the surface velocity $u_{s}$. A local rheology would lead to the following relations:

$$
\bar{u}=\frac{3}{5} u_{s}=\frac{2}{5} \mu^{-1}(\tan \theta) \frac{(g \cos \theta)^{1 / 2}}{d} h^{3 / 2} .
$$

Deviations from a local rheology can therefore be quantified from the measurement of $\bar{u}$ and $u_{s}$. One of the most obvious deviations with respect to the local prediction is the existence of a height $h_{\text {stop }}(\theta)$ below which homogeneous flows eventually stop. Indeed, the local rheology does not contain any length scale that could prescribe $h_{\text {stop }}(\theta)$.

Pioneering experiments have suggested that the Froude number $\bar{u} / \sqrt{g h}$ could be a function of the rescaled flow thickness $h / h_{\text {stop }}(\theta)$. This property is a priori hardly consistent with a local rheology. However, it is observed that $h_{\text {stop }}(\theta)$ diverges as $1 /\left(\tan \theta-\mu_{c}\right)$ and that $I=\mu^{-1}(\tan \theta)$ vanishes as $\tan \theta-\mu_{c}$. For a slope close enough to $\mu_{c}$, the local rheology is therefore consistent with the phenomenological flow rule:

$$
\frac{\bar{u}}{\sqrt{g h}}=\beta \frac{h}{h_{\text {stop }}(\theta)} \text {. }
$$

Experimentally, this relation is observe to hold for glass spheres, of rather well-defined shape and diameter $[9,31]$. However, there are many situations where such a flow rule breaks, for example, for nonspherical grains [9,24,32-34] or very close to the jamming onset [35]. Therefore, a central question is the possible emergence of a nonlocal constitutive relation.

In this article, we will extensively use these elementary predictions of the local rheology to analyze the flow of irregular grains (e.g., rough sand), where significant deviation from the "linear" flow rule [Eq. (3)] was identified [9,24,32-34]. The paper is organized as follows. First, using three different methods, we establish the existence of a jammed layer below a steady avalanching granular flow. We then revisit the flow rule taking into account this result. Thereafter we pursue our exploration of erosion and deposition processes by studying systematically the dynamics and selection mechanisms of solitary waves $[30,32,36,37]$ moving without changing shape on a granular deposit.
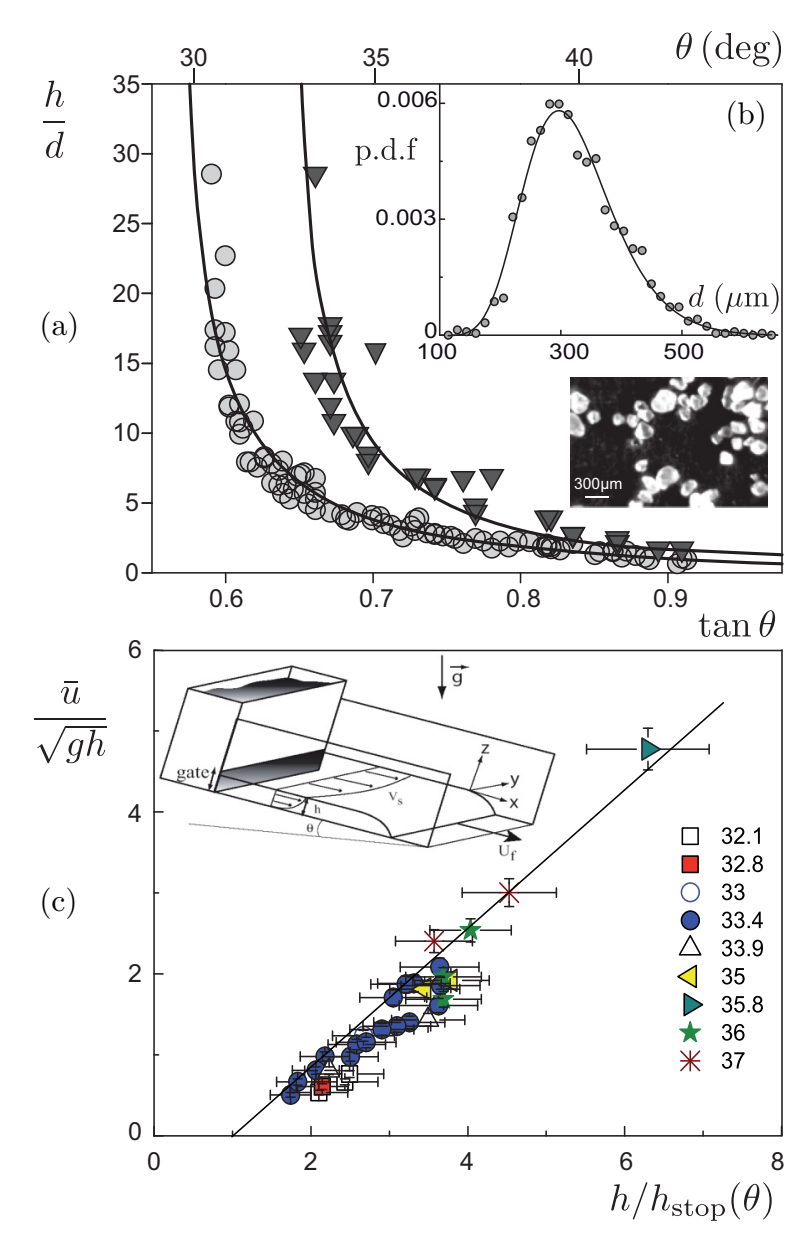

FIG. 1. (Color online) Homogenous flow of sand over a rough substrate. (a) Stability diagram: The circles define the stopping curve $h_{\text {stop }}(\theta) / d$, and the open triangles define the maximal stability curve $h_{\text {start }}(\theta) / d$. The solid lines are fits to the function $\tan \theta=$ $\mu_{c}+\delta \mu /(h / a d+1)$ with $\delta \mu=0.659$ and $a=1.11$. For $h_{\text {stop }}, \mu_{c}=$ 0.555 , and for $h_{\text {start }}, \mu_{c}=0.659$. In the inset (b), we display a picture and the probability density function (p.d.f.) of the grain size (probability over a sample of approximatively 1000 grains), the red curve is a $\log$ normal fit centered around $d=312( \pm 60) \mu$,. (c) The "raw" flow rule derived from the avalanche front velocity, namely the Froude number $\bar{u} / \sqrt{(} g h)$ as a function of the rescaled avalanche height $h / h_{\text {stop }}$. The corresponding incline angles are displayed in the legend. In the inset, we display a schematics of the experimental setup used to obtain the flow rule.

\section{FULLY DEVELOPED FLOW OF ROUGH SAND ON A PLANE}

\section{A. Flow rule}

Systems involving irregular grains (e.g., rough sand) show substantial deviations from the flow rule (3) [9,24,30,32,34]. Here we use Fontainebleau sand of a rather narrow size distribution around $d=312 \pm 60 \mu \mathrm{m}$; see inset of Fig. 1(a). The grains are significantly different from the regular spherical grains usually used in granular experiments [9,31]: in particular, their shape is rough and faceted. The experimental inclined plane is similar to that used by Daerr and Douady [38], with a velvet cloth coating. This system displays a rather wide bistability (hysteretic) domain between the curve $h_{\text {stop }}(\theta)$, 


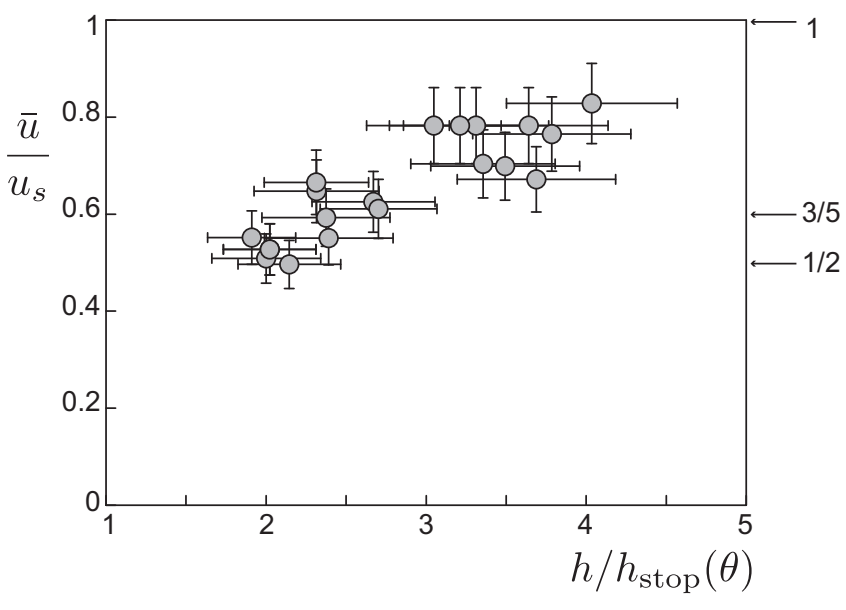

FIG. 2. Velocity ratio $\bar{u} / u_{s}$ as a function of $h / h_{\text {stop }}(\theta)$. Arrows indicate specific velocity ratio $1 / 2,3 / 5$, and 1 , respectively, the linear velocity profile, Bagnold's profile, and the plug flow.

defined above, and the curve $h_{\text {start }}(\theta)$ defined as the minimal granular thickness spontaneously unjamming [Fig. 1(a)].

To derive the sand rheology, systematic experiments were performed by changing the inclined plane angle and the grain flow rate, controlled by the trap aperture. The grain thickness is measured using an inclined laser sheet intersecting the surface, recorded with a video camera (200images/s). The same images are intercorrelated to extract the surface velocity. The mean flow velocity $\bar{u}$ is determined by monitoring the granular front velocity when it passes in front of the camera. The flow rule hence obtained [Fig. 1(c)] shows that within error bars the data points collapse onto a linear master curve, as obtained for most materials. However, contrarily to the case of glass beads, this line does not go through origin. The velocity rather vanishes at $h=h_{\text {stop }}$. Moreover, we do not observe as in the flow rule established by Deboeuf et al. for glass spheres [35] a crossover between a local rheology at large $h$ and a continuous decrease down to $h=h_{\text {stop. It is }}$ worth noting that we could not decrease $h / h_{\text {stop }}$ below 1.8 .

We have measured the ratio of the average velocity $\bar{u}$ by the surface velocity $u_{s}$, which provides a quantitative characteristic of the velocity profile. For a steady flow obeying a local rheology, we recall that $\bar{u} / u_{s}=3 / 5$. For a plug flow one gets $\bar{u} / u_{s}=1$ and for a linear profile, $\bar{u} / u_{s}=1 / 2$. In Fig. 2 we report this ratio as a function of the flow height $h$ rescaled by $h_{\text {stop. }}$. We observe an increase of $\bar{u} / u_{s}$ from 0.5 to 0.8 when $h / h_{\text {stop }}$ is increased from 1.8 to 4 . This is hardly consistent with a local rheology, even at large $h$.

\section{B. Evidence for a jammed layer}

The previous experimental results seem to indicate that for the sand grains we use, we are quite far from the results obtained with glass beads. An important issue is now to figure out whether an essential hypothesis concerning the existence of a fully developed flow of grains holds in this case: is there a jammed layer below the flowing layer?

To investigate this issue, we have used two independent measurement techniques. In both, we have worked as close as possible to steady flow conditions. The constant flow rate is controlled by the opening of the reservoir. The experimental duration of the continuous flow is determined by the limited amount of sand used and is between 50 and 500 s. For the first experiment, a sooted blade was inserted in the flow which induced an erosion of the soot limited to the upper granular layers. After the end of flow, the blade is removed and photographed. Analyzing the image, a sharp transition between eroded and noneroded parts of the sooted blade is clearly visible. Note that we took an explicit care to vary, at a given flow rate, the experimental duration of the flow, and we noticed that in the accessible range of flow durations, the transition height was systematically well defined.

The second set of experiments consist in preparing at a constant angle a deposit of thickness $h_{\text {stop }}(\theta)$. Afterwards, at a downhill distance of about $2 / 3$ of the plane length, we dig down to the bottom a trench spanning about $10 \mathrm{~cm}$ across and $2 \mathrm{~cm}$ along the slope. Then we reconstruct the initial deposit by pouring grains of different colors, layer by layer. Once the trench is filled, the sand flow is started again at a constant flow rate. Thereafter, after submitted to a continuous sand flow, the remaining height of static grains is determined. Both measurement techniques consistently show the presence of a jammed layer (see inset of Fig. 3), at least for an experimental duration of continuous flow between $50 \mathrm{~s}$ and $500 \mathrm{~s}$. Note that quasistatic or creeping layers were observed already in many other experiments (see, e.g., Refs. [12,39-41]), but they

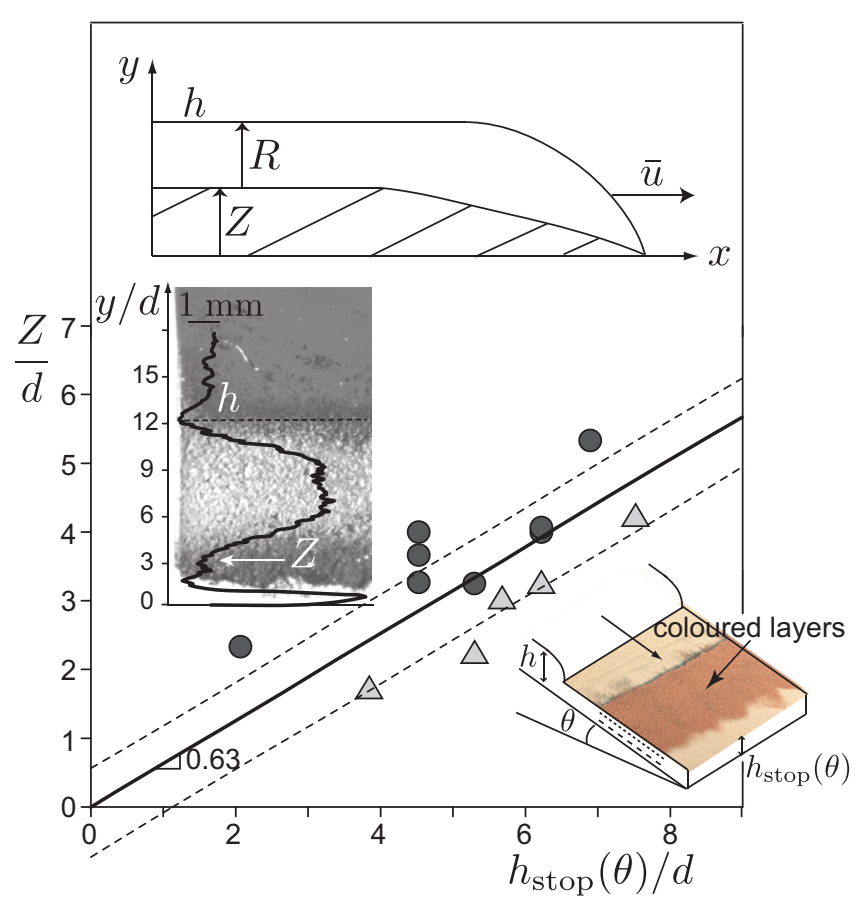

FIG. 3. (Color online) (a) Schematic of the jammed layer during the flow. (b) Jammed layer height $Z / d$ below the flow as a function of the rescaled stopping height $h_{\text {stop }} / d$. Circles correspond to data obtained by the sooted blade technique. Triangles are data obtained with the colored grains layer technique (see text for explanations). The solid line represents the best linear fit for the whole data (i.e., erosion and colored layer techniques) with a slope of 0.63 and forced to zero. The dashed lines are fits of the two independent methods with a fixed slope of 0.63 . 
result from friction on lateral boundaries. The creeping zone is observed in a region below the Coulomb criterion. In the present situation, the stress ratio $\tau / P$ is homogeneous. The static layer comes from the interplay between the rough bottom boundary and possibly the highly irregular shape of the grains. Indeed, in our experiments the avalanche plane had a width about 1200 times the grain diameter $d$, and the resulting granular flows had a maximal height of about $20 \mathrm{~d}$. The jammed layer is between $3 d$ to $8 d$. Therefore, we can neglect the lateral boundary effects as a pertinent mechanism inducing a jammed layer $[12,39,41]$. Within experimental uncertainties, the static layer thickness is well described by a linear relation of the form $Z=0.63 h_{\text {stop }}$.

\section{Sand on sand antidunes}

The presence of a static layer induced by the rough bottom is confirmed by the direct observation of stationary structures below the surface, associated with a modulation of the static layer. We explicitly checked that this phenomenon was not present with round glass beads. To visualize the structures, when the flow height is not too large, we took snapshots of the surface with a time exposure of the order of $1 \mathrm{~s}$. The flowing layer is then blurred, and one clearly distinguishes the modulation by a shadowing effect (see inset of Fig. 4). Using these images, we have computed correlations of the gray-scale profiles along the flow direction and perpendicular to it, and we determined the mean periodicity. The structure wavelength turns out to decrease with the inclination angle (Fig. 4). We do not have any definite explanation for these bed forms that emerge from the erosion and deposition process at work at the interface between the jammed layer and the upper flow. Imagining that there is an analogy with bed forms emerging under the action of a permanent flow, these structures would be antidunes. Indeed, the Froude number is larger than one. This means that gravity effects are lower than inertial effects, allowing for significant modulation of the surface; at a vanishing Froude number, gravity is so important that the free surface must be flat. Like antidunes $[42,43]$, the observed

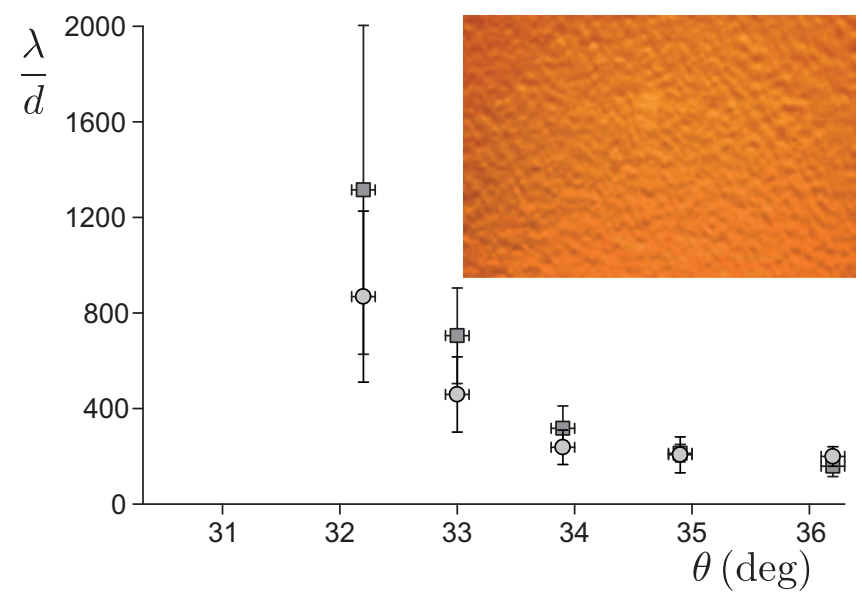

FIG. 4. (Color online) Longitudinal ( $\square$ ) and transverse (०) bedform wavelength $\lambda$ as a function of the inclination angle $\theta ; \theta_{r}$ and $\theta_{a}$ are, respectively, the repose and the avalanche angles measured for the Fontainebleau sand [see also Fig. 1(a)].

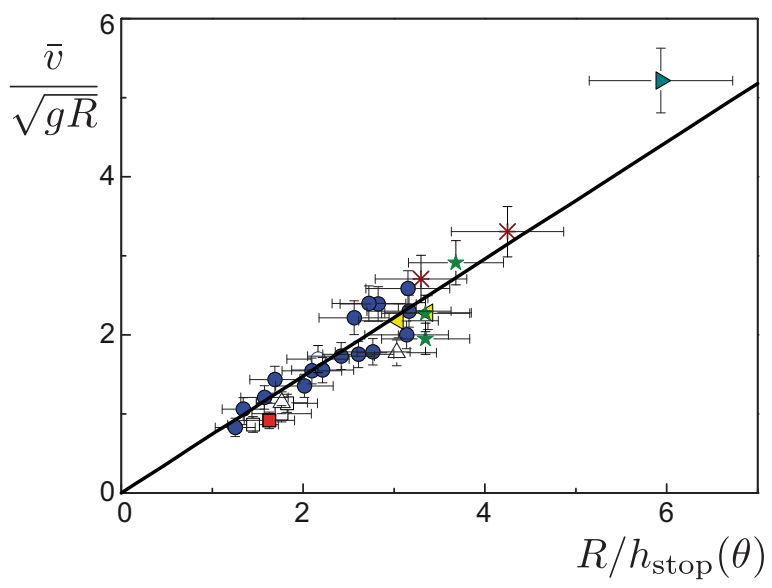

FIG. 5. (Color online) Revisited flow rule. Froude number $\bar{v} / \sqrt{g R}$ as a function of the rescaled flow height $\mathrm{R} / h_{\text {stop }}$ [same data as plotted in Fig. 1(c)]. The full line is the best fit of the equation $y=0.82 x$. We took $R=h-Z$ with $Z=0.63 h_{\text {stop }}(\theta)$ (see Fig. 3).

structures do not visibly propagate over a time comparable to that over which they form. If correct, the analogy would suggest that these structures form by the resonance of surface standing waves excited by the modulation of the static layer.

\section{Flow rule revisited}

Using mass conservation and the fact that grains do not flow in a static layer of thickness $Z$, the velocity averaged over the flowing layer reads

$$
\bar{v}=\frac{h}{R} u_{f} .
$$

Revisiting the flow rule by taking into account the actual flowing layer yields a different relation, consistent with

$$
\frac{\bar{v}}{\sqrt{g R}}=\beta^{\prime} \frac{R}{h_{\text {stop }}(\theta)}
$$

with $\beta^{\prime}=0.74 \pm 0.02$ (see Fig. 5). The linear flow rule is now quite similar to the one found for glass beads down to $R / h_{\text {stop }} \simeq 1.25$ as it seems to pass through zero. Note that in this case, the ratio $R / h_{\text {stop }}$ does not need to be larger than one.

To pursue the test of locality, we also measured the ratio $\bar{v} / u_{s}$ between the velocity $\bar{v}$, averaged over the flow thickness, and the surface velocity. It is plotted in Fig. 6 as a function of $R / h_{\text {stop }}(\theta)$. Within experimental uncertainties, the ratio $\bar{v} / u_{s}$ is around $r=0.87( \pm 0.05)$, which is significantly larger than the value predicted by the local rheology. The velocity profile is therefore more homogeneous than predicted by Bagnold's profile. This could possibly be the signature of a shear band separating a quasiplug flow from the static sublayer.

To go beyond these measurements, one would need the full velocity profile in the bulk of the flow, which is not accessible for such an experiment. In any case our results bring a clear challenging question open for further numerical simulations and theoretical modeling of avalanche flows of rough grains. 


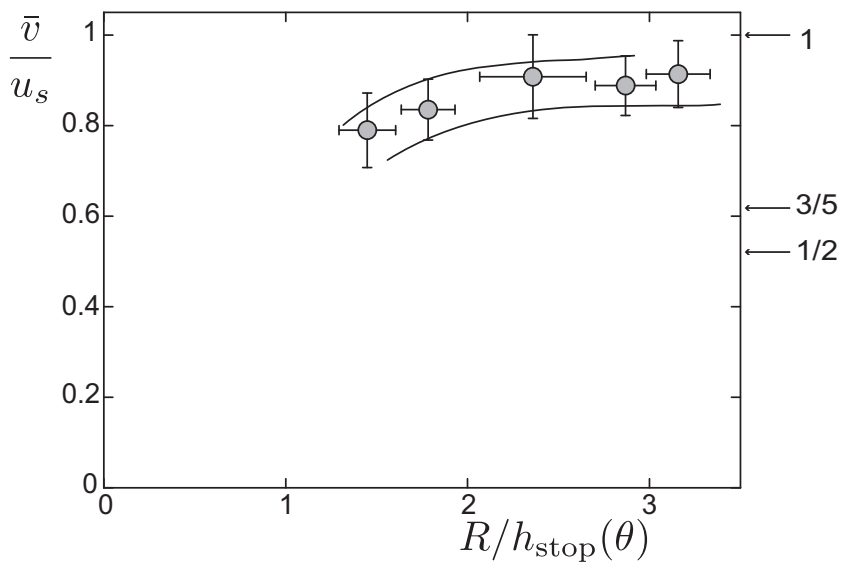

FIG. 6. Ratio $\bar{v} / u_{s}$ of the mean flow velocity to the surface velocity as a function of the rescaled flowing height $R / h_{\text {stop }}$. Dots correspond to data obtained with $Z=0.63 h_{\text {stop }}(\theta)$. Solid lines are for $Z=0.63 h_{\text {stop }}(\theta)+0.56 d$ (erosion method) and $Z=0.63 h_{\text {stop }}(\theta)-$ $0.72 d$ (colored layers method) (see Fig. 3). This gives a good estimation of uncertainties.

\section{EROSION DEPOSITION WAVES OVER AN ERODIBLE SANDY LAYER}

The propagation of avalanches on an erodible layer of grains has already been investigated experimentally for glass beads [30,32,34,38,44] and sand [30,32,36,37]. A particularly interesting family of such avalanches are solitary waves that propagate without changing shape (and mass) [30,33,36,37]. The shape of erosion and deposition avalanches has already been shown to depend very sensitively on the nature of granular particles [30]. In the present situation following Refs. [36,37], we are interested in solitary avalanches, homogeneous in the lateral direction (besides boundary effects) [36,37]. It is a priori different from the situation studied by Borzsonyi et al. [30,33], which features localized three-dimensional avalanches. In order to investigate the dynamics of the interface separating the flowing layer from the static sublayer, we have performed systematic experiments of such "2D" solitary erosion and deposition waves, which propagate, by definition, on a layer of thickness $h_{\text {stop }}(\theta)$. To initiate the avalanche fronts, we designed a "bulldozer" technique where a plate perpendicular to the avalanche track scrapes the sediment at a constant velocity $V_{p}$ over a distance $L_{p}$ (see Fig. 7) on a layer previously prepared at $h_{\text {stop }}(\theta)$ by flowing sand grains continuously for some time and then stopping suddenly the flow.

\section{A. Experimental preparation of a solitary waves}

Solitary waves present an asymmetric "shark-teeth" shape. They are transversally unstable above $33^{\circ}$, and a fingering pattern grows $[36,37,45]$. The analysis presented here is therefore limited to the range of angles for which they are stable. Their velocity $V_{a}$ is rapidly selected and is on the order of $\sqrt{g d} \simeq 5 \mathrm{~cm} / \mathrm{s}$. Figure 7 (a) shows a typical spacetime diagram extracted from a movie of the avalanche at 125 images/s. It is produced by adding sequentially the same pixel line cut along the direction of the flow $x$. We notice that very rapidly the avalanche velocity becomes constant. The plate velocity has little influence on the avalanche shape
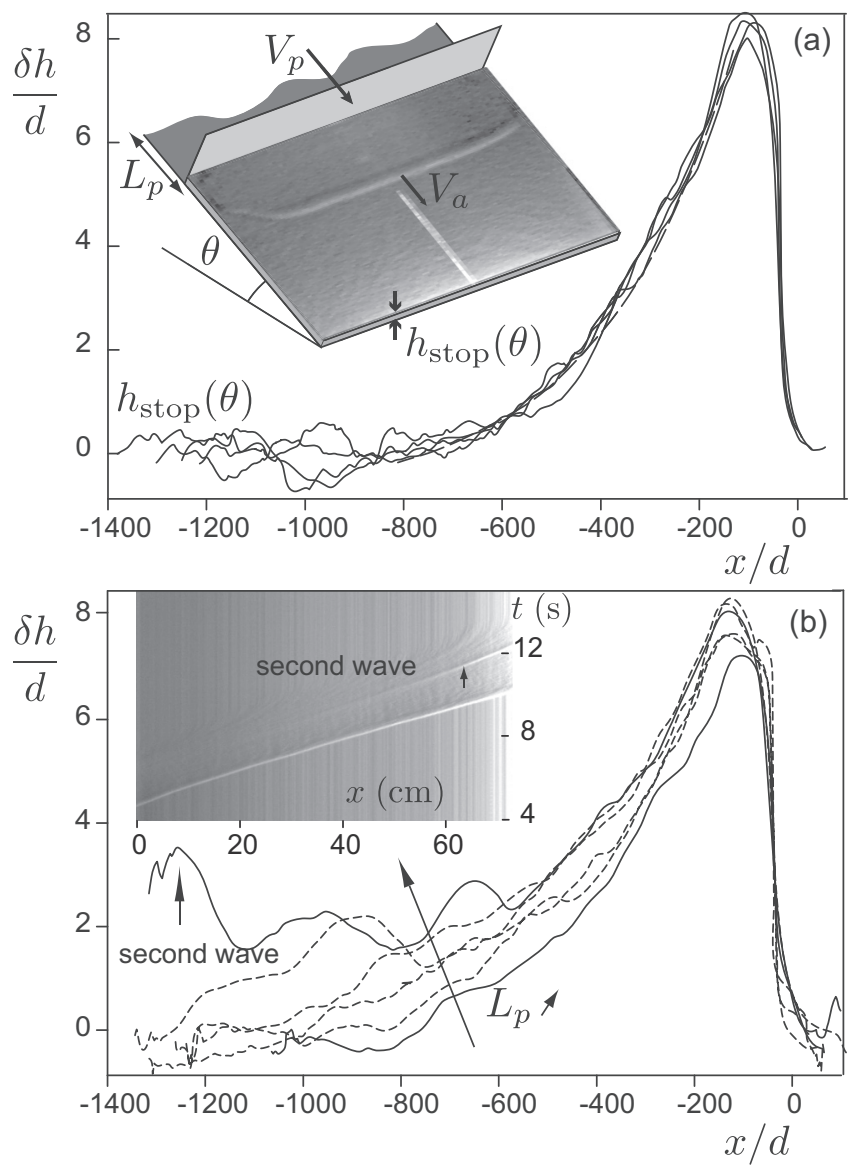

FIG. 7. Inset to (a): Production of solitary waves using the scraping experimental procedure described in the text. (a) Solitary wave velocity $V_{a}$ and avalanche profiles for increasing scraping velocities $V_{P}=2,3,4,6,7 \mathrm{~cm} / \mathrm{s}\left(\theta=32^{\circ}\right)$. Inset to (b): Spatiotemporal diagram showing the emergence of a second avalanche when the scraping lenght $L_{P}=40 \mathrm{~cm}$ is large $\left(V_{P}=4 \mathrm{~cm} / \mathrm{s}\right)$; The arrows indicates the position of the two fronts. (b) Avalanche profiles for increasing scraping lengths $L_{P}=8,10,12,14,16,18,20,40 \mathrm{~cm}$ (increasing arrow), $V_{P}=4 \mathrm{~cm} / \mathrm{s}$. The vertical arrow shows the onset of a secondary avalanche occurring for a scraping length $L_{P} \geqslant 16 \mathrm{~cm}$.

[Fig. 7(a)] and on its final velocity $V_{a}$. In the majority of the experiments, we therefore kept this parameter at a typical value of $\sqrt{g d} / 3$. On the other hand, the scraping length changes the mass of grains trapped in the body of the avalanche. Figure 7(b) shows that the shape varies with $L_{P}$. The tail of the solitary wave becomes larger and relaxes back to $h_{\text {stop }}$ more slowly when $L_{p}$ increases. When the amount of grains set initially into motion is too large, the avalanche splits into two (or more) pieces.

Note that it is not possible to deduce the mass of moving grains from the sole measurement of the avalanche profiles. Its dependence with the mass per unit length of scrapped grains $m=\rho L_{P} h_{\text {stop }}$ (the effective density is around $\rho=1600 \mathrm{~kg} / \mathrm{m}^{3}$ ) is a priori a difficult issue since we need to know the jammed layer position below the avalanche. To get a direct information on this quantity, we have collected at the edge of the plane, in a vessel of fixed width $W$, the moving mass of grains. The area of rolling grains trapped in 


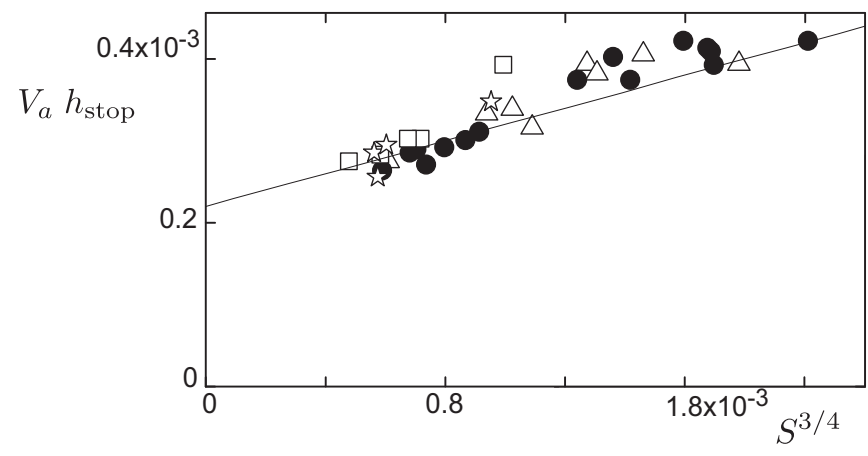

FIG. 8. Selection of the avalanche velocity $V_{a}$ as a function of the area of rolling grains trapped $S$ and several inclination angle $\theta$.

the avalanche is deduced as $S=M /(\rho W)$. As shown in Fig. 8 this quantity is nicely related to the avalanche velocity $V_{a}$ (while $L_{P}$ is not). For different scrapping lengths (at constant angle $\theta$ ), the velocity is an increasing function of $S$.

\section{B. Dynamics of the solitary erosion and deposition waves}

We now use transversally homogeneous solitary waves to get insight into the dynamics of erosion and deposition processes. First, we test to which extend the measured velocities and the shape profiles correspond to a situation where inertia is important. To this purpose, we estimate the magnitude of the different terms involved in the momentum equation of Navier-Stokes. The grain velocity magnitude are typically on the order of $\sqrt{g d}$. The heights $h$ or $R$ can roughly be estimated around $5 d$, which yields a typical value for the flow rate $\approx 5 \sqrt{g d} d$. In the frontal part of the avalanche $\partial_{x} \approx 1 / 10 d$ and in the rear part $\partial_{x} \approx 1 / 1000 d$. The flow is in a steady state in the frame of reference of the avalanche. The acceleration is therefore negligible. The inertial transport of momentum is around $g / 10$ at the front and $g / 1000$ in the rear. The shear stress is set by the basal friction at the bottom of the flowing layer and vanishes at the surface. The shear stress gradient is therefore around $g$. Inertia can therefore be neglected and the lubrication approximation applied. The pressure gradient is controlled by the free surface slope and is on the order of $\rho g$ at the front and $\rho g / 100$ in the rear part, which can be considered as "locally homogeneous."

The analysis of the surface velocity and the corresponding avalanche profile exhibit three distinct regions (Fig. 9): the front, where the pressure gradient is important $(-300<$ $x / d<0)$, the avalanche core $(-1600<x / d<-300)$, for which height and velocity are related, and finally a tail region, $-2100<x / d<-1600$, where $h \approx h_{\text {stop }}$ is reached and only about one layer of grains is still rolling on the surface. The different regions really manifest themselves quantitatively in Fig. 10 showing $F r$ vs $h / h_{\text {stop }}$. The core (in thick line) lies in a linear continuation of the points obtained using the full avalanche measurements. It starts when $h / h_{\text {stop }} \approx 1$ up to a crossover region where $\operatorname{Fr}\left(h / h_{\text {stop }}\right)$ decreases. Quantitatively, the separation between the core and the front is chosen at the maximum of the Froude number. The tail corresponds to the region where the Froude number decreases rapidly for $h / h_{\text {stop }} \approx 1$. The tail is defined as the zone were the rolling grains do not cover the whole surface any more. The flow

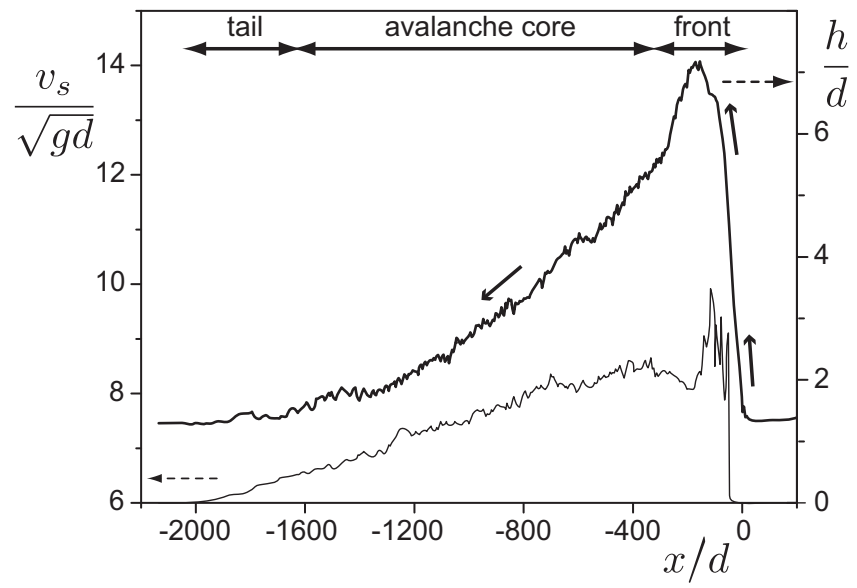

FIG. 9. Solitary avalanche profiles. (a) Superimposed experimental profiles of the rescaled avalanche height (right) $h / d$ and surface Froude number (left) $v_{s} / \sqrt{g h}\left[\theta=32^{\circ}\right.$, trapped mass onset $m_{c}=8900( \pm 200) d^{2}$, avalanche velocity $\left.V_{a}=1.9( \pm 0.1)(g d)^{1 / 2}\right]$. The solitary wave is composed of three parts: the front, where inertia is dominant, and the avalanche core and the tail, where inertia is negligible. The arrows correspond to a displacement along the avalanche from front to tail.

ceases to be homogeneous along the transverse direction, and we observe for this $h / h_{\text {stop }} \approx 1$ condition patches of flowing grains becoming less and less frequent as the avalanche passes by. This reveals a very fragile state characterizing the transition between a liquid and a solid. These are general and robust features of such an erosion-deposition wave. The shark-teeth profile bears a striking similarity with the $3 \mathrm{D}$ localized avalanche shape studied by Borzonyi et al. [30,32]

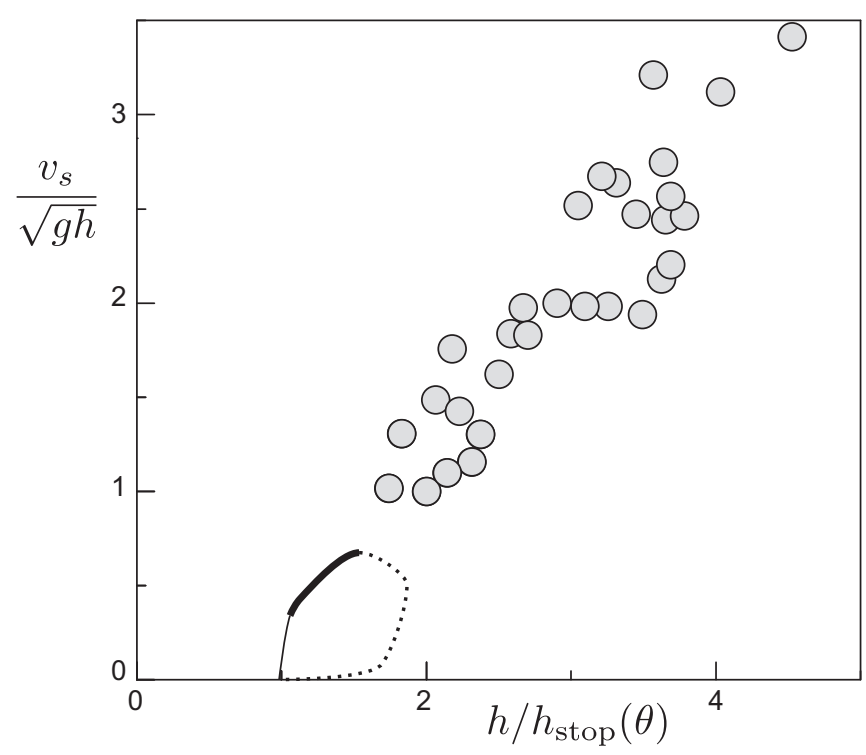

FIG. 10. Flow rule for steady state flows Fig. 1 and for a solitary wave (angle shown $\theta=32^{\circ}$ ). Surface Froude number $v_{s} / \sqrt{g h}$ as a function of the rescaled avalanche height: $h / h_{\text {stop. The thick line }}$ corresponds to the core of the solitary avalanche, the thin line to the tail, and the dotted line to the front. The flow rule for the core of the solitary avalanche is in continuity with the flow rule of the steady state flow. 
for rough grains (when the profiles are cut in their symmetry plane). For example, it appears that in both cases the velocity front slightly leads the height front.

Interestingly, in spite of the fact that inertial effects can be neglected for the average flow, the stoppage conditions are nontrivial. Like for stationary homogeneous flows, it is interesting to plot the Froude number $u_{s} / \sqrt{g d}$, based on the surface velocity $u_{s}$ measured along the solitary wave, as a

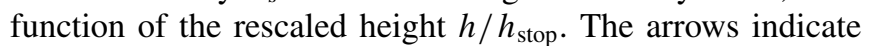
the displacement of the avalanche from front to tail. When $h$ approaches $h_{\text {stop }}$ the mean surface velocity remains finite in the avalanche core and drops suddenly in the tail (see Fig. 9). This behavior corresponds to a progressive trapping of the rolling grains on the surface. Note that the time scale needed for the flow to stop is quite large with respect to the "natural" trapping time $(g / d)^{1 / 2}$. In Fig. 10 we report the flow rule both for the steady uniform flows and for the solitary waves. For uniform flow, the mean flow velocity $\bar{u}$ has been changed in surface velocity by using the relation obtained for Fig. 2: $\bar{u} / u_{s} \simeq 0.26+0.14 h / h_{\text {stop }}$. From this figure we see that the core of solitary waves obeys the same flow rule as steady state flows: the solitary wave dynamics allows one to extend the investigation of the erosion and deposition processes down to $h=1.1 h_{\text {stop }}$.

\section{SUMMARY AND PERSPECTIVES}

In this paper we have focused on the rheological properties of a dense flow of sandy material flowing on a avalanche plane with a rough substrate. We specifically studied the erosion and deposition processes associated to the fully developed steady flow and to the dynamics of transversally stable solitary waves. In the first part, we established a relation between the Froude number and the ratio between the layer height and the stopping height. We evidence a grouping of the data for different fluxes and different flowing angles. However, this flow rule is quite different from the one put forward with well-characterized round grains: it is a linear relation that does not go through zero. This result seems to be the hallmark of many avalanche experiments using rough grains, which are found to deviate significantly from what is observed for spherical grains [9,24,32-34], which, like numerical simulations, are closer to the ideal. In this case, using two different experimental techniques, we evidence a jammed layer forming below the flowing part, allowing us to reformulate the flow rule using the flowing layer height only. This result should be put in perspective with the fact that we use a quite dissipative substrate (the velvet cloth). It would be interesting to see if this result still holds with a harder, rough boundary (as, for example, in Refs. [33,34]). In the second part, we have studied transversally homogeneous solitary waves propagating on an erodible substrate which is the jammed layer at rest at the stopping height. We investigated in detail the selection mechanism for the flowing mass and the avalanching velocity. We also show that the dynamics of the solitary wave can help to get insight into the dynamics of erosion and deposition processes very close to the jamming onset.

\section{ACKNOWLEDGMENTS}

B.A. is supported by Institut Universitaire de France. This work is funded by the ANR JamVibe and a CNES research grant.
[1] F. Charru, B. Andreotti, and P. Claudin, Ann. Rev. Fluid Mech. 45, 469 (2013).

[2] M. S. Papa, S. Egashira, and T. Itoh, Natl. Hazards Earth Sys. Sci. 4, 469 (2004).

[3] R. M. Iverson, M. Logan, and R. P. Denlinger, J. Geophys. Res. 109, F01015 (2004).

[4] L. Hsu, W. E. Dietrich, and L. S. Sklar, J. Geophys. Res. 113, F02001 (2008).

[5] B. Sovilla and P. Bartelt, Nat. Hazard Earth Syst. Sci. 2, 169 (2002).

[6] J. E. Simpson, Ann. Rev. Fluid Mech. 14, 213 (1982).

[7] J. L. R. Allen, Developments in Sedimentology: Sedimentary Structures (Elsevier, Amsterdam, 1984).

[8] P. S. Dodds and D. H. Rothman, Annu. Rev. Earth Planet Sci. 28, 571 (2000).

[9] G. D. R. Midi (collective work), Eur. Phys. J. E 14, 341 (2004).

[10] F. da Cruz, S. Emam, M. Prochnow, J.-N. Roux, and F. Chevoir, Phys. Rev. E 72, 021309 (2005).

[11] Somewhat similar formulation of the rheology for dense granular flows was suggested earlier by J. D. Goddard, Acta Mech. 63, 3 (1986).

[12] P. Jop, Y. Forterre, and O. Pouliquen, Nature (London) 441, 727 (2006).

[13] J. T. Jenkins and S. B. Savage, J. Fluid Mech. 130, 187 (1983).

[14] B. Andreotti and S. Douady, Phys. Rev. E 63, 031305 (2001).
[15] B. Andreotti, Eur. Phys. Lett. 79, 34001 (2007).

[16] J. T. Jenkins, Phys. Fluids 18, 103307 (2006).

[17] A. Tanguy, F. Leonforte, and J.-L. Barrat, Eur. Phys. J. E 20, 355 (2006).

[18] A. Lemaître and C. Caroli, Phys. Rev. Lett. 103, 065501 (2009).

[19] L. Bocquet, A. Colin, and A. Ajdari, Phys. Rev. Lett. 103, 036001 (2009).

[20] E. Lerner and I. Procaccia, Phys. Rev. E 79, 066109 (2009).

[21] A. Amon, V. B. Nguyen, A. Bruand, J. Crassous, and E. Clément, Phys. Rev. Lett. 108, 135502 (2012).

[22] E. Lerner, G. Düring, and M. Wyart, Proc. Natl. Acad. Sci. USA 109, 4798 (2012).

[23] I. S. Aranson and L. S. Tsimring, Phys. Rev. E 64, 020301(R) (2001); 65, 061303 (2002).

[24] I. S. Aranson, L. S. Tsimring, F. Malloggi, and E. Clement, Phys. Rev. E 78, 031303 (2008).

[25] D. Volfson, L. S. Tsimring, and I. S. Aranson, Phys. Rev. E 68, 021301 (2003).

[26] I. S. Aranson and L. S. Tsimring, Rev. Mod. Phys. 78, 641 (2006).

[27] K. Kamrin and G. Koval, Phys. Rev. Lett. 108, 178301 (2012).

[28] D. L. Henann and K. Kamrin, Proc. Natl. Acad. Sci. USA 110, 6730 (2013). 
[29] M. Bouzid, M. Trulsson, P. Claudin, E. Clément, and B. Andreotti, Phys. Rev. Lett. 111, 238301 (2013).

[30] T. Börzsönyi, T. C. Halsey, and R. E. Ecke, Phys. Rev. E 78, 011306 (2008)

[31] O. Pouliquen, Phys. Fluids 11, 542 (1999).

[32] T. Börzsönyi, T. C. Halsey, and R. E. Ecke, Phys. Rev. Lett. 94, 208001 (2005).

[33] T. Börzsönyi and R. E. Ecke, Phys. Rev. E 76, 031301 (2007).

[34] D. Takagi, J. N. McElwaine, and H. E. Huppert, Phys. Rev. E 83, 031306 (2011).

[35] S. Deboeuf, E. Lajeunesse, O. Dauchot, and B. Andreotti, Phys. Rev. Lett. 97, 158303 (2006).

[36] F. Malloggi, J. Lanuza, B. Andreotti, and E. Clément, in Powders \& Grains edited by R. Garcia-Rojo, H. J. Herrmann, and S. McNamara (Balkema, Rotterdam, 2005), p. 997; F. Malloggi, J. Lanuza, B. Andreotti, and E. Clément, Europhys. Lett. 75, 825 (2006).
[37] I. S. Aranson, F. Malloggi, and E. Clément, Phys. Rev. E 73, 050302(R) (2006).

[38] A. Daerr and S. Douady, Nature (London) 399, 241 (1999).

[39] T. S. Komatsu, S. Inagaki, N. Nakagawa, and S. Nasuno, Phys. Rev. Lett. 86, 1757 (2001).

[40] R. Delannay, M. Louge, P. Richard, N. Taberlet, and A. Valance, Nat. Mater. 6, 99 (2007).

[41] N. Taberlet, P. Richard, A. Valance, W. Losert, J. M. Pasini, J. T. Jenkins, and R. Delannay, Phys. Rev. Lett. 91, 264301 (2003).

[42] B. Andreotti, P. Claudin, O. Devauchelle, O. Durán, and A. Fourrière, J. Fluid Mech. 690, 94 (2012).

[43] A. Recking, V. Bacchi, M. Naaim, and P. Frey, J. Geophys. Res. 114, F04025 (2009).

[44] A. Mangeney, O. Roche, O. Hungr, N. Mangold, G. Faccanoni, and A. Lucas, J. Geophys. Res. 115, F03040 (2010).

[45] E. Clement, F. Malloggi, B. Andreotti, and I. S. Aranson, Granular Matter 10, 3 (2007). 